\title{
DEVELOPMENT OF COPROCESSED EXCIPIENTS OF XANTHAN GUM AND ACACIA GUM AS A CONTROLLED RELEASE MATRIX FOR FAMOTIDINE FLOATING TABLETS
}

\author{
UNSYURA DHIPA BUDAYA ${ }^{1,2}$, SILVIA SURINI ${ }^{1 *}$ \\ ${ }^{1}$ Laboratory of Pharmaceutics and Pharmaceutical Technology Development, Faculty of Pharmacy, Universitas Indonesia, Depok, West \\ Java, Indonesia. 'Department of Production, Drs. Mochamad Kamal Pharmaceutical Division of Indonesia Navy, Jakarta, Indonesia. \\ Email: silvia@farmasi.ui.ac.id
}

Received: 26 September 2019, Revised and Accepted: 17 December 2019

ABSTRACT

Objective: Controlled release floating tablets require excipients, which act as a matrix to control the release of the active drug and facilitate the tablet floating in the gastric milieu. One potential excipient is coprocessed excipients of xanthan gum and acacia gum (Co-XG-GA), which is a physical modification of the two natural polymers. In this study, we produced several Co-XG-GA and used them as matrices in floating tablet formulations.

Methods: Several coprocessed excipients were prepared from xanthan gum and acacia gum at ratios of 1:1, 1:2, 2:1, 1:3, and 3:1. The obtained excipients were then characterized physically, chemically, and functionally. The coprocessed excipients were then formulated in floating tablets using famotidine as the drug model. The floating tablets were then evaluated in terms of the tablet floating capabilities and the drug release in $\mathrm{HCl}$ medium at $\mathrm{pH} 1.2$ for $8 \mathrm{~h}$.

Results: Our results showed that the coprocessed excipients were a fine powder, odorless, and a grayish-white color. The excipients had a good swelling index, fairly large viscosity, and good gel strength; hence, they were suitable to be applied as the matrices of floating tablet formulations. The floating tablets of F2, which contained the Co-XG-GA 1:2, demonstrated the best characteristics with $8.33 \pm 0.58$ min of floating lag time and $24 \mathrm{~h}$ of total floating time. Further release studies revealed that the famotidine floating tablets, which used Co-XG-GA (F1-F5) as matrices, controlled drug release with zero-order release kinetics and could be used for controlled release dosage forms.

Conclusion: Collectively, our results indicate that the Co-XG-GA can be applied as matrices in controlled release floating tablets.

Keywords: Acacia gum, Coprocessed excipient, Famotidine, Floating tablet, Xanthan gum.

(C) 2020 The Authors. Published by Innovare Academic Sciences Pvt Ltd. This is an open access article under the CC BY license (http://creativecommons. org/licenses/by/4. 0/) DOI: http://dx.doi.org/10.22159/ijap.2020.v12s1.FF044

\section{INTRODUCTION}

Pharmaceutical preparations with gastroretentive drug delivery system are one of the ongoing developments to overcome absorption and bioavailability problems that often occur in ordinary oral dosage forms. This is partAicularly true for drugs with large absorption in certain areas of the digestive tract. Floating drug delivery systems (FDDSs) are delivery systems based on preparations that float in the gastric fluid due to their lower densities compared with gastric fluid. Accordingly, these preparations remain buoyant in the stomach for a long period without affecting the gastric emptying rate [1,2].

There are various types of natural gums, including those derived from natural polymers such as the natural polysaccharides, xanthan gum and acacia gum. Xanthan gum has good flow properties and is considered stable and non-toxic. In addition, it is a good gelling and viscosityincreasing agent (viscosity enhancer). Xanthan gum also has high swelling properties due to large water absorption and slight erosion [3]. However, xanthan gum has the disadvantage that it is not a tablet binder. In contrast, acacia gum is a stable material, a good gelling agent, and behaves like a tablet binder, but it has swelling properties with low viscosity. However, acacia gum is unique because it forms colloidal solutions with low viscosity compared with other gums. To obtain better overall characteristics of the two excipients, a modification of the coprocessed excipients of xanthan gum and acacia gum (Co-XG-GA) was performed. Modification of the coprocess was performed by combining the two excipients in the form of solution dispersion using a codrying process. The purposes of the coprocess excipient were to increase synergy and functionality, change the physicochemical properties, and cover the shortcomings of each of its constituent excipient [4,5]. The coprocess excipient obtained from the xanthan gum and acacia gum was then used as a matrix in floating tablet formulations.

Famotidine, which is one of the drugs absorbed in the stomach, was used as the drug model in this study. Famotidine is a histamine 2 (H2) receptor blocker with low bioavailability, ranging from $40 \%$ to $45 \%$ on oral administration. In addition, famotidine has an elimination halflife of 2.5-4 h. Drugs such as famotidine are suitable for preparation in the FDDS because they allow delivery to the $\mathrm{H} 2$ receptors found in gastric parietal cells. Famotidine in the form of FDDS serves to reduce the known side effects of famotidine, including diarrhea, dizziness, headache, and anorexia. This is because famotidine is in a sustained release formulation, thereby reducing fluctuations in blood drug levels.

\section{METHODS}

\section{Materials}

Famotidine was gifted by Molex Ayus Co. Ltd. (Indonesia). Xanthan gum was purchased from Cargill Bioengineering (Canada). Acacia gum was purchased from Shangqiu Kangmedia Bio-Tech (China). Other chemicals and solvents were of analytical grade and purchased from commercial suppliers.

\section{Preparation of Co-XG-GA}

Xanthan gum and acacia gum, at concentrations of 3\% each, were dispersed in distilled water. The xanthan gum and acacia gum were 
mixed at varying ratios $(1: 1,1: 2,2: 1,1: 3$, and $3: 1)$ and homogenized with a rotation of $3000 \mathrm{rpm}$ for $30 \mathrm{~min}$ to obtain a homogeneous mass. The mass was then dried with a double drum drier at $80 \pm 5^{\circ} \mathrm{C}$. The obtained layer or flakes were milled and sieved using a 35 mesh. Chemical, physical, and functional characterizations of the mass were then performed.

\section{Functional group analysis}

Chemical characterization was performed by functional group analysis using infrared spectrophotometers. Several Co-XG-GA were weighed, mixed until homogeneous with dried $\mathrm{KBr}$ powder, and then scanned in the region of wavenumbers $4000-400 \mathrm{~cm}^{-1}$.

\section{Particle size distribution}

Particle size distribution was determined using a sieve method. The sieve was arranged from the smallest mesh of 35 mesh, followed by 45 mesh, 60 mesh, 80 mesh, 120 mesh, and the largest at 230 mesh. The sample (25 g) was sieved with the smallest mesh at $30 \mathrm{rpm}$ for 20 min. Each sieve that contained powder was weighed. Then, the weight percentage of the powder was displayed on a particle size distribution diagram.

\section{Thermal analysis}

Physical characterization was performed by thermal analysis using a differential scanning calorimeter (DSC). Approximately $5 \mathrm{mg}$ of the sample was placed into an aluminum cylinder with a diameter of $5 \mathrm{~mm}$. The cylinder was covered with aluminum plates and then the sample was put into a DSC. Measurements were examined at $30-35^{\circ} \mathrm{C}$ with a heating rate of $10^{\circ} \mathrm{C} / \mathrm{min}$. Endothermic and exothermic processes that occurred in the sample were recorded [6].

\section{Swelling index of floating tablets}

The swelling index was performed using the powder excipient molded into a tablet with a weight of approximately $500 \mathrm{mg}$. The Petri dish and initial tablet $\left(\mathrm{W}_{1}\right)$ were weighed. Each tablet was placed into a Petri dish containing $10 \mathrm{~mL}$ of $\mathrm{HCl}$ at $\mathrm{pH}$ 1.2. After $15 \mathrm{~min}$, the $\mathrm{HCl}$ was removed from the Petri dish and carefully dried, and the tablet and Petri dish were weighed to obtain the weight of the hydrated tablets $\left(\mathrm{W}_{2}\right)$. The same weighing procedure was performed at intervals of $30,60,120,180,240$, $360,480,600$, and $720 \mathrm{~min}$. The time taken during the weighing process was not counted as a time interval. The volume of the $\mathrm{HCl}$ medium must remain at $10 \mathrm{~mL}$ at the time of the swelling index to the next interval [7].

\section{Gel strength test}

The powder was dispersed in aquadest at a concentration of $10 \% \mathrm{w} / \mathrm{v}$ and heated at $120^{\circ} \mathrm{C}$ to form a gel. Measurements were performed using a texture analyzer (Rheoner 3305, Germany). The gel preparation was placed into the sample container and then the penetration device was lowered to the surface of the gel. The gel strength was then measured when the gel breaks.

\section{Preparation of famotidine floating tablets}

For the preparation of the famotidine floating tablets, the wet granulation method was adopted. Polymer powder, famotidine, and $\mathrm{NaHCO}_{3}$ were mixed until homogeneous. After that, talc, Mg stearate, and Avicel PH-101 were added and then molded manually using a tablet press machine. The composition of each formula is shown in Table 1.

\section{Floating test}

The tablets were placed in a $100 \mathrm{~mL}$ glass beaker containing simulated gastric fluid, $\mathrm{pH} 1.2(\mathrm{HCl} 0.1 \mathrm{~N})$ at $37^{\circ} \mathrm{C}$. The time required for the tablet to rise to the surface and to float was taken as the floating lag time (FLT). The duration of time in which the tablet constantly remained on the surface was considered as total floating time [8].

\section{Famotidine content estimation in tablets}

Three tablets of each formula were used to test for the drug content. Each tablet was crushed to a powder, weighed, and then placed into a $100 \mathrm{~mL}$ volumetric flask and dissolved with $0.1 \mathrm{~N} \mathrm{HCl}$. After that, the solution was filtered and diluted suitably. The absorbance of the resultant solution was then measured with ultraviolet (UV) spectrophotometer at a wavelength of $265 \mathrm{~nm}$. The amount of drug contained in the sample was calculated with the help of a calibration curve from a standard solution [6].

\section{Dissolution study}

Dissolution apparatus type 1 (the basket type) was used for the dissolution study. A drug release study was performed using $900 \mathrm{~mL}$ $0.1 \mathrm{~N} \mathrm{HCl}$ at $37 \pm 0.5^{\circ} \mathrm{C}$ with a speed of $50 \mathrm{rpm}$. Sampling $(10 \mathrm{~mL})$ was performed at $15,30,60,120,180,240,360,480,600$, and $720 \mathrm{~min}$. The amount of solution taken was replaced by an equal amount of 0.1 $\mathrm{N} \mathrm{HCl}$ solution. The absorbance of the sample was measured with a UV spectrophotometer at a wavelength of $265 \mathrm{~nm}$. The drug release profile was determined by plotting the percentage of drug released against time. The dissolution data obtained were then calculated to determine the kinetic model of drug release and the mechanism of release.

\section{Drug release mechanism}

The mechanism of drug release from the preparations was determined by plotting the dissolution data in kinetic models of zero order, first order, Higuchi equation, and Korsmeyer-Peppas equation, as shown in Table 2 [9].

\section{RESULTS AND DISCUSSION}

\section{Preparation of Co-XG-GA}

The percentage yields of the Co-XG-GA were $40-70 \%$, as shown in Table 3. Weight loss occurred during the process of dispersing the

Table 1: Formulas of famotidine floating tablets

\begin{tabular}{lllllllll}
\hline Material & F1 & F2 & F3 & F4 & F5 & F6 & F7 & F8 \\
\hline Famotidine & 40 & 40 & 40 & 40 & 40 & 40 & 40 & 40 \\
Co-XG-GA (1:1) & 300 & - & - & - & - & - & - & - \\
Co-XG-GA (1:2) & - & 300 & - & - & - & - & - & - \\
Co-XG-GA (2:1) & - & - & 300 & - & - & - & - & - \\
Co-XG-GA (1:3) & - & - & - & 300 & - & - & - & - \\
Co-XG-GA (3:1) & - & - & - & - & 300 & - & - & - \\
Physical mixture of & - & - & - & - & - & 300 & - & - \\
XG-GA & & & & & & & & \\
Xanthan gum (XG) & - & - & - & - & - & - & 300 & - \\
Acacia gum (GA) & - & - & - & - & - & - & - & 300 \\
NaHCO (15\%) & 75 & 75 & 75 & 75 & 75 & 75 & 75 & 75 \\
Talc (2\%) & 10 & 10 & 10 & 10 & 10 & 10 & 10 & 10 \\
Mg stearate (1\%) & 5 & 5 & 5 & 5 & 5 & 5 & 5 & 5 \\
Avicel 101 & 70 & 70 & 70 & 70 & 70 & 70 & 70 & 70 \\
\hline
\end{tabular}

Co-XG-GA: Coprocessed excipients of xanthan gum and acacia gum

Table 2: Drug release kinetic models

\begin{tabular}{ll}
\hline Model & Equation \\
\hline Zero order & $\mathrm{Q}_{\mathrm{t}} / \mathrm{Q}_{0}=\mathrm{k}_{0} \mathrm{t}$ \\
First order & $\ln \mathrm{Q}_{\mathrm{t}} / \mathrm{Q}_{0}=\mathrm{k}_{1} \mathrm{t}$ \\
Higuchi & $\mathrm{Q}_{\mathrm{t}} / \mathrm{Q}_{0}=\mathrm{k}_{\mathrm{H}} \mathrm{t}^{1 / 2}$ \\
Korsmeyer-Peppas & $\mathrm{Q}_{\mathrm{t}} / \mathrm{Q}_{0}=\mathrm{kt}^{\mathrm{n}}$ \\
\hline
\end{tabular}

$\mathrm{Q}_{t} / \mathrm{Q}_{0}$ : The drug fraction that is released at time $\mathrm{t}, \mathrm{k}_{0}, \mathrm{k}_{1}, \mathrm{k}_{\mathrm{H}}, \mathrm{k}$ : The drug release constants for each equation, $\mathrm{n}$ : Peppas diffusion exponent

Table 3: Yield in preparation of Co-XG-GA excipients

\begin{tabular}{ll}
\hline Excipients & Yield (\%) \\
\hline Co-XG-GA (1:1) & 61.67 \\
Co-XG-GA (1:2) & 53.33 \\
Co-XG-GA (2:1) & 71.67 \\
Co-XG-GA (1:3) & 48.33 \\
Co-XG-GA (3:1) & 76.67 \\
\hline
\end{tabular}

Co-XG-GA: Coprocessed excipients of xanthan gum and acacia gum 


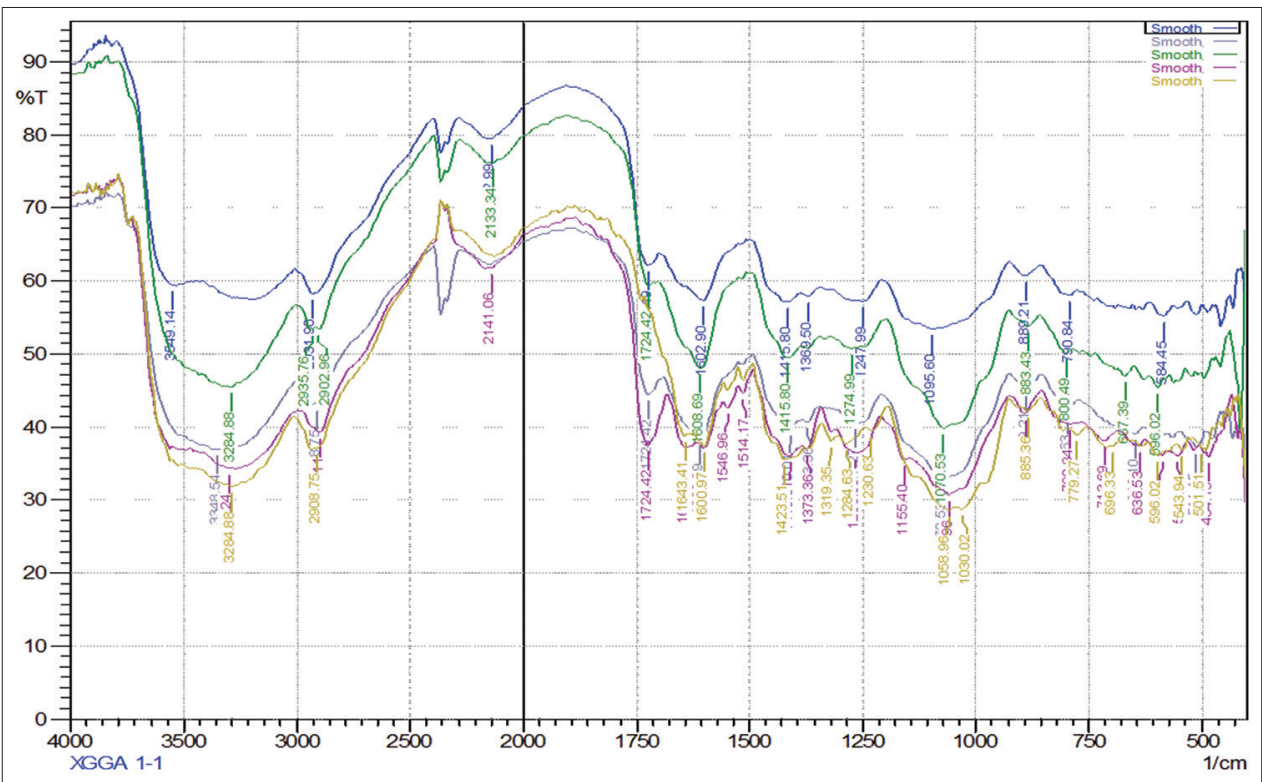

Fig. 1: Infrared spectrum of excipients: (a) Xanthan gum, (b) acacia gum, (c) coprocessed excipients of xanthan gum and acacia gum (COXG-GA) 1:1, (d) CO-XG-GA 1:2, and (e) CO-XG-GA 2:1

excipients in distilled water, along with material left behind during the drying, milling, and sifting process

\section{Functional group analysis}

The purpose of the functional group analysis was to ascertain whether a chemical reaction occurred between each of the functional groups of the two excipients. Xanthan gum and acacia gum only interact or mix physically. No new functional groups presented in the spectrum (Fig. 1), indicating that these interactions do not involve chemical changes. The Fourier-transform infrared spectroscopy spectrum of xanthan gum, at wavenumber $1724.42 \mathrm{~cm}^{-1}$ showed a sharp peak, suggesting the presence of $-\mathrm{C}=\mathrm{O}$ carboxylic bonds. This is reinforced by the presence of wide peaks with strong intensities in the range of wavenumbers $2400-3700 \mathrm{~cm}^{-1}$, indicating the presence of the $-\mathrm{OH}$ carboxylate group.

\section{Particle size distribution}

The particle size distributions of the coprocessed excipients are shown in Fig. 2. The excipients of Co-XG-GA 1:1, Co-XG-GA 1:2, Co-XG-GA 2:1, Co-XG-GA 1:3, and Co-XG-GA 3:1 were distributed on the particle size of $64-125 \mu \mathrm{m}$, with the percentages at $40.00 \%, 48.70 \%, 27.03 \%, 44.62 \%$, and $26.27 \%$, respectively. Particle size can affect the flowability of a powder: The smaller the particle size of the powder, the more difficult it is to flow, or it shows poor flow properties due to high interparticle cohesiveness.

\section{Thermal analysis}

Fig. 3 displays thermal analysis of the Co-XG-GA using DSC (PerkinElmer, USA). The results show that the Co-XG-GA 1:1, 1:2, and 2:1 excipients were first degraded at temperatures $<100^{\circ} \mathrm{C}$. According to the previous research, polysaccharides first degrade at a temperature of $40-110^{\circ} \mathrm{C}$. Degradation at this temperature was associated with heat loss. Physical interactions involving high-temperature heating causes thermal stability to decrease [10]. The physical interaction of the polymer causes the formation of two descending peaks on the curve.

\section{Swelling index}

The results of the swelling index of the famotidine floating tablets are shown in Fig. 4. The Co-XG-GA excipient could swell for $12 \mathrm{~h}$ in $\mathrm{HCl} \mathrm{pH}$ 1.2. The Co-XG-GA 3:1 (F5) excipient had the highest swelling index at $307.35 \%$, whereas the Co-XG-GA 1:3 (F4) excipient had the lowest swelling index at 131.16\%. The Co-XG-GA 1:1 (F1), 1:2 (F2), and 2:1 (F3) excipients had swelling indexes of $193.87 \%, 150.47 \%$, and $270.63 \%$, respectively.

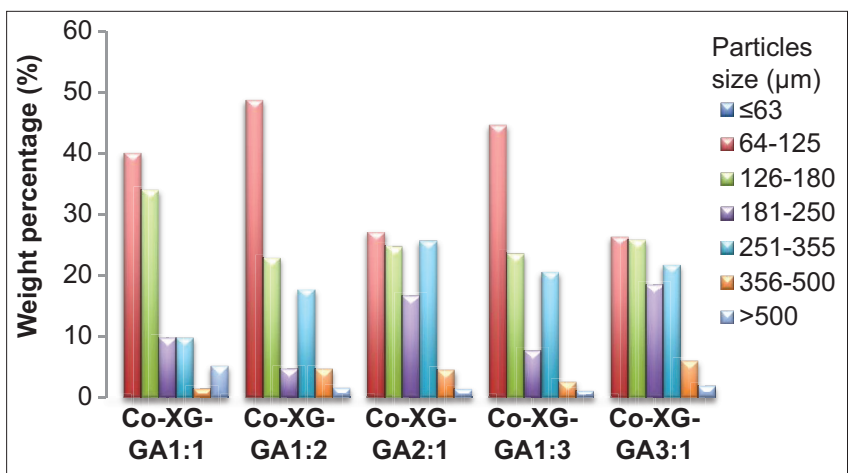

Fig. 2: Particle size distribution of coprocessed excipients of xanthan gum and acacia gum (Co-XG-GA) 1:1, Co-XG-GA 1:2, Co-XGGA 2:1, Co-XG-GA 1:3, and Co-XG-GA 3:1

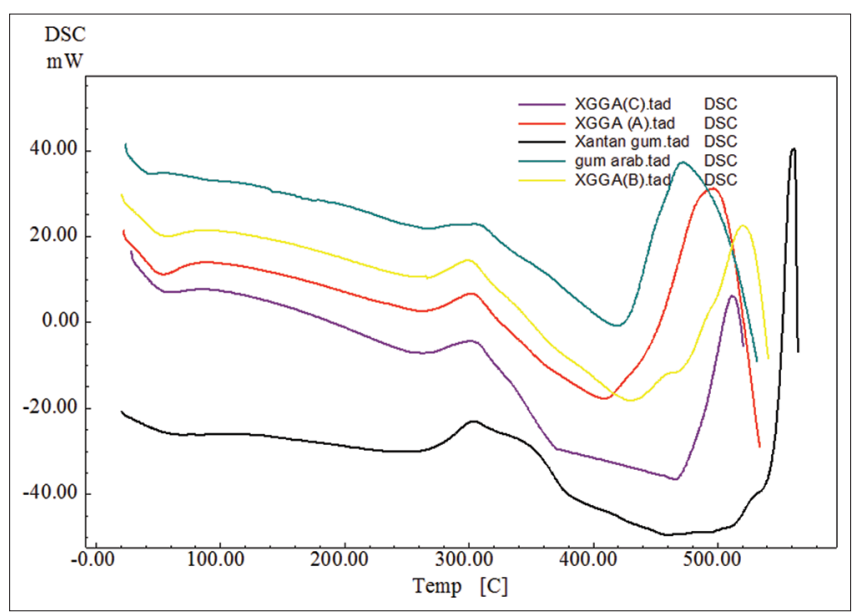

Fig. 3: Endothermic and exothermic curves: (A) Coprocessed excipients of xanthan gum:acacia gum (Co-XG: GA) 1:1, (B) CoXG: GA 2:1, (C) Co-XG: GA 1:2, (D) XG, and (E) GA

Gel strength test

Observation data on the strength of the excipient gel are shown in Table 4. The gel strength is the resistance of a gel to external pressure 
Table 4: Gel strength of the excipients

\begin{tabular}{ll}
\hline Material & Gel strength (gF) \\
\hline Co-XG: GA 1:1 & $17.5 \pm 0.87$ \\
Co-XG: GA 1:2 & $14.67 \pm 0.58$ \\
Co-XG: GA 2:1 & $32.67 \pm 1.53$ \\
Co-XG: GA 1:3 & $7.83 \pm 0.29$ \\
Co-XG: GA 3:1 & $36.17 \pm 0.76$ \\
Physical mixture & $13.83 \pm 0.29$ \\
Xanthan gum (XG) & $55.67 \pm 0.76$ \\
Acacia gum (GA) & $0 \pm 0$ \\
\hline
\end{tabular}

Co-XG: GA: Coprocessed excipients of xanthan gum: acacia gum

Table 5: Floating lag time and total floating time of the famotidine floating tablets composed of the Co-XG-GA

\begin{tabular}{lll}
\hline Formula & FLT (min) & TFT (h) \\
\hline F1 & $8.67 \pm 0.58$ & 24 \\
F2 & $8.33 \pm 0.58$ & 24 \\
F3 & $13.33 \pm 0.58$ & 24 \\
F4 & $10.33 \pm 0.58$ & 24 \\
F5 & $15.67 \pm 0.58$ & 24 \\
F6 & $18.33 \pm 0.58$ & 24 \\
F7 & $8.67 \pm 0.58$ & 24 \\
F8 & $0.00 \pm 0$ & 0 \\
\hline
\end{tabular}

Co-XG-GA: Coprocessed excipients of xanthan gum and acacia gum, FLT: Floating lag time, TFT: Total floating time

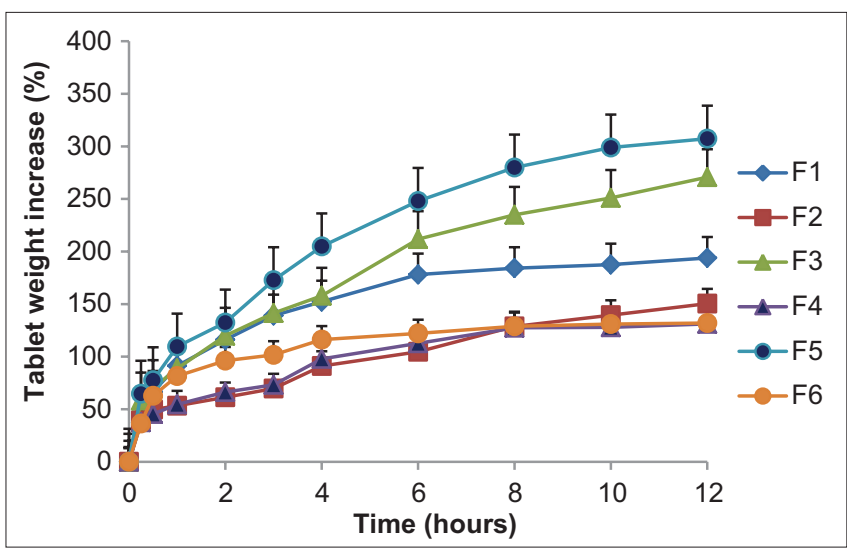

Fig. 4: Swelling index of the F1-F5 floating tablets for $12 \mathrm{~h}$ in $\mathrm{HCl}$ pH 1.2, F6 as a comparison. Each point represents the mean value $\pm S D(n=3)$

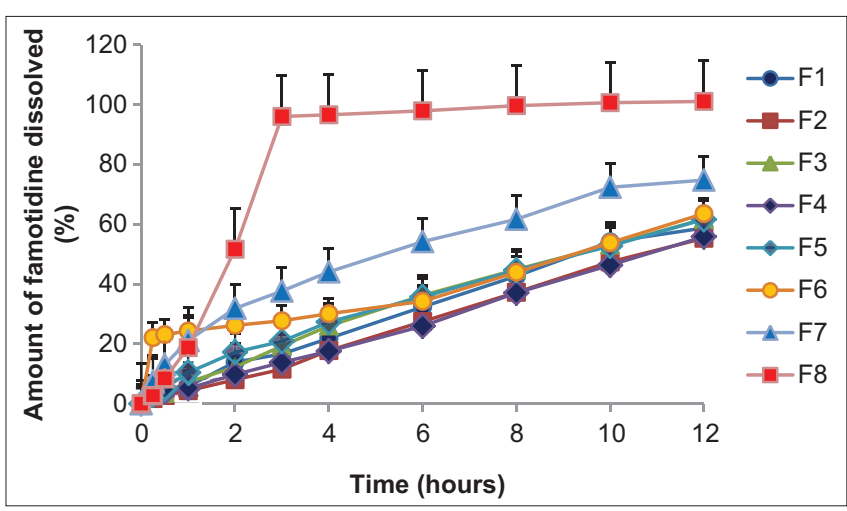

Fig. 5: The release profile of the famotidine floating tablets of F1-F5 formulation in $\mathrm{HCl}$ medium of $\mathrm{pH} 1.2$ for $12 \mathrm{~h}, \mathrm{~F} 6-\mathrm{F} 8$ as a comparison. Each point represents the mean value \pm standard deviation $(n=3)$
Table 6: Evaluation results of famotidine content in the floating tablets

\begin{tabular}{ll}
\hline Formula & Content (\%) \\
\hline F1 & $97.50 \pm 1.18$ \\
F2 & $102.56 \pm 1.17$ \\
F3 & $105.61 \pm 0.25$ \\
F4 & $101.21 \pm 1.12$ \\
F5 & $106.33 \pm 0.97$ \\
F6 & $99.54 \pm 1.48$ \\
F7 & $100.33 \pm 0.90$ \\
F8 & $99.29 \pm 1.44$ \\
\hline
\end{tabular}

Table 7: Drug release rate constant (k) of the famotidine floating tablets

\begin{tabular}{lll}
\hline Formula & \multicolumn{2}{l}{ Kinetics parameters } \\
\cline { 2 - 3 } & $\mathbf{k}\left(\mathbf{h}^{-\mathbf{1}}\right)$ & $\mathbf{r}$ \\
\hline F1 & $0.050 \pm 0.003$ & 0.9969 \\
F2 & $0.046 \pm 0.001$ & 0.9980 \\
F3 & $0.050 \pm 0.001$ & 0.9960 \\
F4 & $0.045 \pm 0.002$ & 0.9980 \\
F5 & $0.048 \pm 0.002$ & 0.9940 \\
\hline
\end{tabular}

and shows the nature of the cohesiveness of a gel in maintaining its shape.

\section{Floating test}

From the results of the floating test shown in Table 5, the F2 tablets composed of Co-XG-GA 1:2 excipient as the matrix had the fastest FLT value at 8.33 min. In contrast, the F5 tablets composed of Co-XGGA excipient 3:1 as a matrix had the longest FLT value of $15.67 \mathrm{~min}$. The best formula was the F2 formula, which contained the Co-XG-GA $1: 2$ excipient matrix on each tablet, and this formula had the fastest floating time among the five formulas at $8.33 \pm 0.58 \mathrm{~min}$. This is because the Co-XG-GA 1:2 excipient was a combination of the natural polymers, which had a swelling index of $150.47 \%$ and a gel strength $14.67 \mathrm{gF}$; thus, it could absorb water immediately when in contact with the $\mathrm{HCl}$ at $\mathrm{pH} 1.2$ medium [11]. This served to wet the gas-forming portion so that the formation of carbon dioxide gas was faster, which causes the preparation to float faster, and the resulting carbon dioxide gas can be retained by the Co-XG-GA 1:2 excipient matrix.

\section{Famotidine content in the floating tablets}

The famotidine content in the tablets is shown in Table 6 and is in the range of $90.0-110.0 \%$ and thereby meeting the requirements of the United States Pharmacopoeia $30^{\text {th }}$ edition.

\section{Drug release study}

Fig. 5 shows the drug release profiles of the famotidine floating tablets. It shows that all the formulas that used a matrix of the coprocessed excipients could sustain drug release for $12 \mathrm{~h}$ with the cumulative amount released: F1 58.75\%, F2 55.32\%, F3 61.75\%, F4 55.86\%, F5 $61.65 \%$, F6 63.59\%, F7 74.75\%, and F8 101.08\%. The release profile of the famotidine floating tablets of formulas $1-5$ followed by the zeroorder kinetics model and the drug release rate constants $(\mathrm{k})$ is shown in Table 7. In this zero-order kinetics model, the drug release profile from the dosage form had a constant release rate from time to time without being affected by the concentration of the drug in the dosage form. This release kinetics model is also ideal for a controlled release dosage form because it can produce constant drug levels in the blood.

\section{CONCLUSION}

In this study, we produced the Co-XG-GA at ratios of 1:1, 1:2, 2:1, 1:3, and $3: 1$ in the form of odorless and slightly grayish-white fine powders. The 
Co-XG-GA excipients had a characteristic swelling index and viscosity that were quite large, with gel strength of 7.83-36.17 gF; thus, this excipient was considered favorable for use in floating matrix tablets. The best formula was the second formula (F2), which contained a 1:2 Co-XG-GA excipient matrix on each tablet and had the fastest floating time among the five formulas at $8.33 \pm 0.58 \mathrm{~min}$, with drug release for $8 \mathrm{~h}$ at $37.26 \%$. All floating tablets with a Co-XG-GA excipient matrix showed a controlled release of famotidine over $24 \mathrm{~h}$.

\section{CONFLICTS OF INTEREST}

The authors declare no conflicts of interest.

\section{REFERENCES}

1. Arora S, Ali J, Ahuja A, Khar RK, Baboota S. Floating drug delivery systems: A review. AAPS PharmSciTech 2005;6:E372-90.

2. Khan F, Ibn Razzak SM, Khan ZR, Azad MA, Chowdhury JA, Reza S. Theophylline loaded gastroretentive floating tablets based on hydrophilic polymers: Preparation and in vitro evaluation. Pak J Pharm Sci 2009;22:155-61.

3. Shaikh A, Shaikh P, Pawar Y, Kumbhar S, Katedeshmukh R. Effect of gums and excipients on drug release of ambroxol $\mathrm{HCl}$ sustained-release matrices. J Curr Pharm Res 2010;6:11-5.
4. Chougule AS, Dikpati A, Trimbake T. Formulation development techniques of co-processed excipients. J Adv Pharm Sci 2012;2:231-49.

5. Surini S, Ariani L, Putri KS, Hayun H. Coprecessed excipients of crosslinked amylose and xanthan gum for use in controlled release dosage forms. Int J App Pharm 2018;10:59-65.

6. The United States Pharmacopoeia Convention. United States Pharmacopoeia $32^{\text {nd }}$ and National Formulary $27^{\text {th }}$. Rockville: The USP Convention; 2009.

7. Bigucci F, Luppi B, Cerchiara T, Sorrenti M, Bettinetti G, Rodriguez L, et al. Chitosan/pectin polyelectrolyte complexes: Selection of suitable preparative conditions for colon-specific delivery of vancomycin. Eur J Pharm Sci 2008;35:435-41.

8. Kumar R, Patil MB, Patil SR, Paschapur MS. Formulation and evaluation of effervescent floating tablet of famotidine. Int J Pharm Tech Res 2009; 1:754-63.

9. Siepmann J, Peppas NA. Modeling of drug release from delivery systems based on hydroxypropyl methylcellulose (HPMC). Adv Drug Deliv Rev 2001;48:139-57.

10. Carbinatto FM, de Castro AD, Cury BS, Magalhães A, Evangelista RC. Physical properties of pectin-high amylose starch mixtures cross-linked with sodium trimetaphosphate. Int J Pharm 2012;423:281-8.

11. Patil MS, Vidyasagar G, Patil VB. Formulation, optimization and evaluation of floating tablets clarithromycin. Int J Pharm Pharm Sci 2015;7:320-6. 